\title{
Rethinking Morphological Analysis Application for Concept Synthesis in Engineering Design
}

\author{
By Jan Erik Heller \\ Manuel Loewert \\ Joerg Feldhusen ${ }^{+}$
}

In a globalised world, enterprises are forced to deal with challenging market conditions. Customers' demand for individualised products increases and de-sign processes need to be faster and cost efficient. Engineering departments are forced to bring up new approaches to remain competitive. Hence, systematic innovation is vital for success. One way to address concept generation is the analysis of functions and their division into sub-functions, for which several principle solutions can be designed or taken from catalogues. Those individual solutions can be combined with the help of morphological boxes to obtain so-called overall solutions. Although widely published, this method is not well-established in industrial application and often misused in academia: it does not prevent bad decisions. The large number of possible overall solutions resulting from combinatorial explosion is still not manageable. A literature review is conducted to understand the initial aim of morphological analyses. Two contradicting directions can be observed: the search for solutions either towards innovation potential or towards technical feasibility. Both come with drawbacks. To overcome these shortages, a new method is presented intending to support engineers. It is founded on the hypothesis that taking context information into account reduces the overall effort. This leads to an iterative approach with gradually substantiating applications of low-complexity morphological boxes. Mathematical concepts like pareto-efficiency are integrated to optimise the multiplicity resulting from combination. An accompanying software tool is presented. Concluding, a discussion of both method and tool in an application example for next generation machine tool concept elaboration is conducted.

Keywords: Morphological analysis, Morphological box, Product concept synthesis, Systematic engineering design process.

\section{Preliminaries}

Companies are increasingly confronted with challenging market conditions resulting from globalisation. Their customers desire more individualised products which is reflected in trends like mass customisation (Pine, 1993). Engineering

\footnotetext{
* PhD Student, RWTH Aachen University, Germany.

$\star$ Chief Engineer, RWTH Aachen University, Germany.

${ }^{+}$Professor, RWTH Aachen University, Germany.
} 
design has to adjust its development processes if it wants to stay competitive. One key factor to achieve this is systematic innovation applied within early phases of the product emergence process. Therefore, it is a prerequisite for entrepreneurial success (Wheelwright and Clark, 1992), (Pahl et al., 2007).

In order to support innovation processes on the engineering side, multiple methods have been conceived. One popular approach analyses the overall function, which is further structured into sub-functions (VDI Guideline 2221). This procedure is conducted to split up the initial problem into manageable subproblems. Afterwards, several possible solutions are set up for each sub-function to cover the complete solution field. Nevertheless, like all methods that split up larger problems into smaller ones, the solutions have to be integrated to build one overall solution fulfilling the initially defined purpose of the product. Consequently, a synthesis step is required. Morphological boxes are used in many cases to assemble overall concepts by systematically linking the individual solutions (Koller, 1998), (Pahl et al., 2007). The procedure for this general approach of turning product ideas into product concepts is given in Figure 1.

Figure 1. Procedure for Systematic Product Conception Product Idea

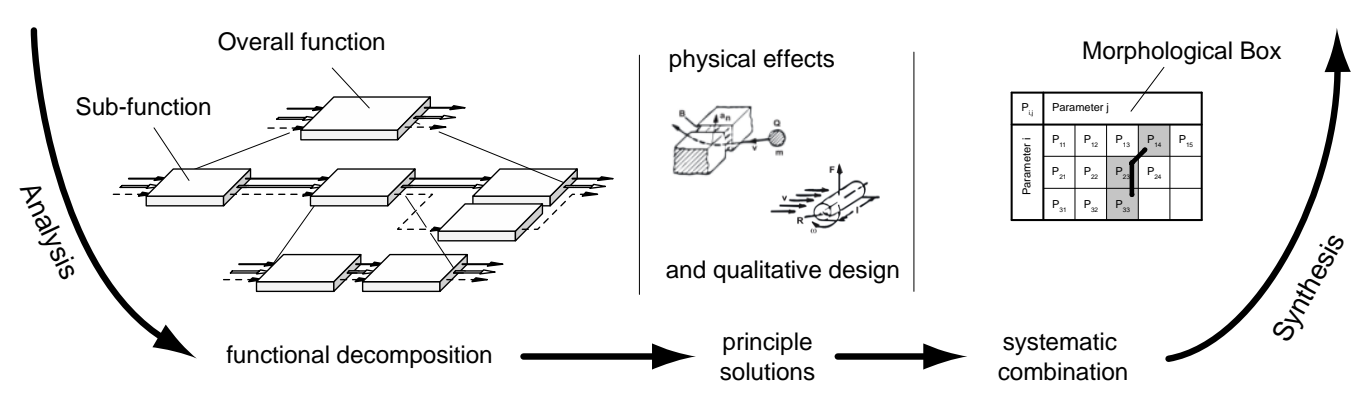

While this method is thoroughly published, it is often not well-implemented in industrial application and frequently improperly utilised in academia. Characteristically, it does not help to avoid making wrong decisions. Moreover, the morphological box can easily be used to justify the designers' preferred solutions without covering the intended complete solution space (Tomiyama et al., 2009). Absurdly, this makes the application of the method useless. In addition to that, methods combining elements suffer from so called combinatorial explosion. This expression refers to the high number of overall solutions that the systematic combination of all single elements creates. Some methods have been published, addressing this challenge. However, none of them can resolve the problem of the high effort in a feasible way for industrial application and at the same time being able to cover a complete solution field. Nevertheless, since a systematic examination of the complete solution field is still promising, new approaches have to be developed.

State of the art of morphological analyses constitutes the main topic of the second section of this paper. The third section investigates optimisation approaches aiming at making combinatorial explosion manageable within product development processes. A new method that overcomes the presented challenges is 
presented before discussing a prototypical software solution. The paper concludes with a summary and an outlook.

\section{Morphological Analyses in Theory}

Morphological analysis does not originate from the discipline of design methodology itself and its application is not limited to this area. Swiss physicist Fritz Zwicky published the fundamental thoughts in the at around the middle of the twentieth century (Zwicky, 1947). He can be considered the initiator of the morphological theory. According to Zwicky, it comprises both analysis and synthesis of comprehensive solution spaces for specific problems (Zwicky, 1949). Hereby, the approach involves the open-minded examination of all theoretically thinkable solutions. He introduces a couple of morphological methods one of which is the method of the morphological box.

\section{The Method of the Morphological Box}

The foremost instrument of the morphological analysis is the morphological box. It is a visual illustration of the morphological field. A box with three dimensions is displayed in Figure 2 (left, a)). The means of presentation used there is the drawer visualisation. The morphological field is displayed as a block consisting of drawers. Each box of every drawer represents one combined overall solution consisting of one element from every dimension. One overall solution is highlighted in the example (in a) as well b)) that consists of the individual elements $P_{14}, P_{23}$ and $P_{33}$.

Figure 2. Morphological Boxes Displayed with Different Means of Presentation
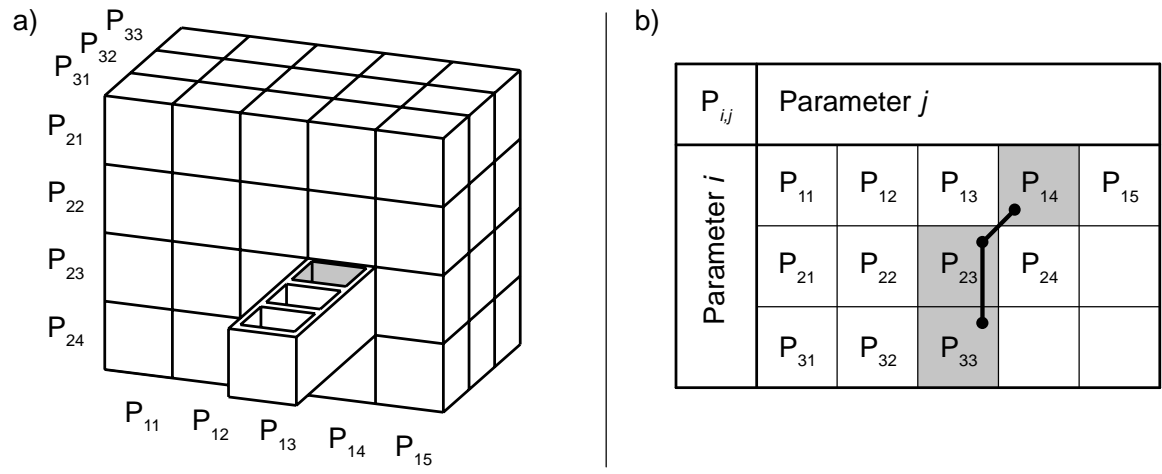

While the drawer presentation enables easy understanding of the morphological box as a concept, it can only be used for solutions from one to three dimensions, as the imaginable coordinate space is limited. However, this presentation can be used to obtain a first impression of the possible amount of overall solutions. Opposed to that, the matrix representation is able to display an infinite number of dimensions (Figure 2 right, $b$ ). 


\section{Applications in Engineering Design}

The method of the morphological box is applicable in many cases within engineering design methodology. The expression is often used synonymously for systematic variations. In this case, the box is used for tabular arrangement of all single solutions to graphically combine possible overall solutions. It is then often referred to as ordering scheme. The individual sub-functions of the product are arranged in the first column of the chart (Pahl et al., 2007). Correspondingly, various working principles or function carriers (Bernhardt, 1981) are arranged in the cells of the respective rows. Picking exactly one element from each row at a time creates product concepts. Roth proposes to put tasks instead of functions in the first column and solutions into the cells (Roth 2000) and then synthesize product concepts. Several catalogues with solutions are available for different tasks (Roth, 2000), (Koller and Kastrup, 1998), (VDI 2727).

Birkhofer researched analysis as well as synthesis of technical products in his thesis, in particular with the help of the morphological box (Birkhofer, 1980). In addition to its purpose as a tool for systematic combination, the morphological box enables simple documentation for the various concepts. Rough or detailed sketches for principle solutions can easily be inserted into template charts.

Tomiyama reviews several methods of different authors and compiles an overview. Specifically, he arguments that the approach presented by Pahl et al. although it might be the most taught method - could easily be misused by engineering design students as well as professionals. Operators might use the morphological box to justify their personally preferred and in many cases intuitively found solutions instead of having followed to the intended procedure for the morphological analysis, that is to say, to investigate the complete solution field. (Tomiyama et al., 2009)

\section{Challenges and Optimisations for Morphological Analyses}

Franke presents the axiom of systematic engineering design. He postulates the need to always investigate every possible solution and thus cover the complete solution space in order to find the best solution (Franke, 1999). This approach resembles the procedure presented by Zwicky (1949). Presumably, this complete investigation is not implementable for enterprises due to their economic boundary conditions. Moreover, in most cases it is not only not needed but also unhelpful to investigate all solutions. From an economic attitude, it is sufficient to elaborate one working solution fitting manufacturing, assembling and other company constraints. However, it is vital to find this solution with the first attempt. These requirements result in a contradiction with the application of morphological analyses. Several approaches are published that try to overcome this unsatisfactory situation. They can be separated into two main categories: first, the ones that are using morphological analyses as creativity techniques. These methods aim at conceiving innovative solutions or try to expand the investigation into areas that were previously not within focus. The second category comprises 
the ones that use morphological boxes for the synthesis of innovative product concepts. With them, the expansion of the solution field is not primarily addressed. Rather the systematic combination of existing components in order to identify at least one viable solution is intended. The two categories can be interpreted as two axes of a coordinate system as given in Figure 3. The image displays two hypothetic investigations with morphological analyses. Both are shown as black squares. With increasing distance from the origin on the y-axis the analysis covers a more extensive solution space. Therefore, knowledge used as well as effort required is increased with each single solution that is added to the investigation. Likewise, the farer to the right an investigation is plotted on the $\mathrm{x}$ axis the more concrete the solutions can be considered regarding their technical feasibility. Again, the effort grows with increasing distance from the origin.

Figure 3. Optimisation Directions for Morphological Analyses

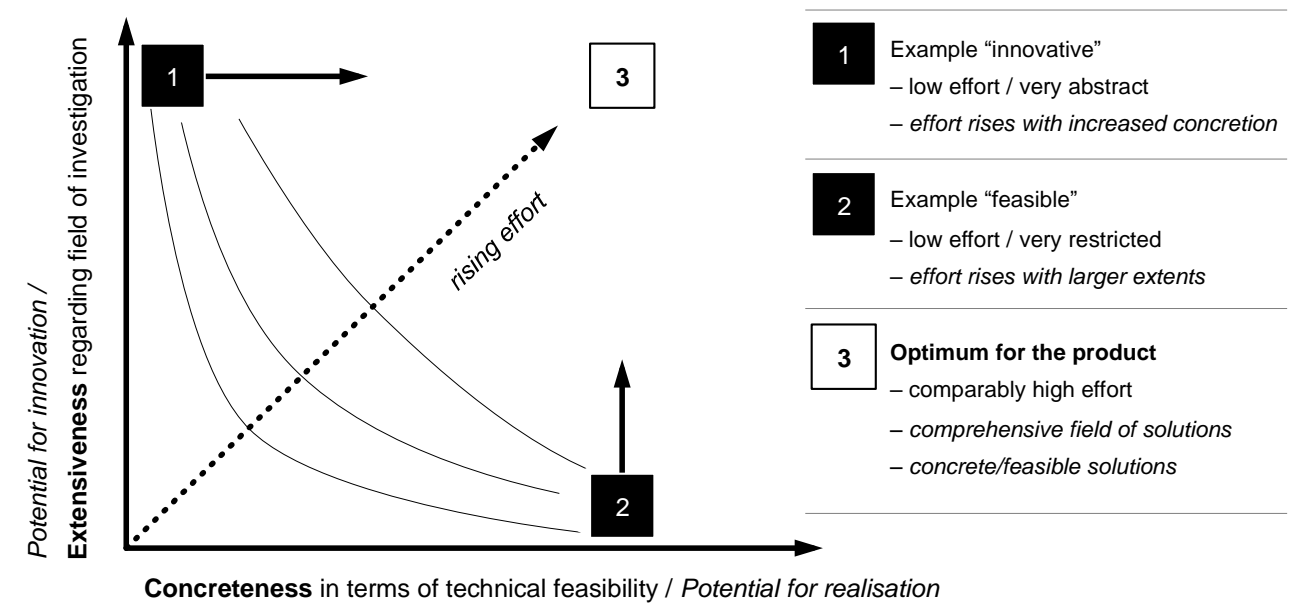

Thus, ideal for new product development would be to both increase the extensiveness of the solution field covered by the research and to choose individual solutions as concrete as possible. This will lead to an investigation that would be plotted in the upper right of the chart. However, the effort to undertake a morphological analysis with these constraints will bring with it an insurmountable effort. Thus, most approaches optimise the investigation towards either better extensiveness or better concreteness.

\section{Preconditions for Optimisations}

All approaches that focus on the efficient management of morphological boxes have in common that they require two theoretical constructs. The first is a priority evaluation for all individual solutions resulting in a ranking. The second is a compatibility matrix that examines the principle possibility to combine two or more individual solutions with each other. Birkhofer presents the constructs as sub-complexes $N_{n}$. He addresses the priority evaluation as complex $N_{1}$ whereas the compatibility evaluation as sub-complex $N_{2}$ (Birkhofer, 1980). 
The quantity of all individual solutions comprising the overall solution space is addressed with the first sub-complex $N_{1}$. This number resembles the quantity of elements in the morphological box. If $x$ refers to the number of rows of the morphological box and $k_{i}=j$ denotes the number of elements within the $i$-th row then the values $r_{i, j}$ represent the respective ranks of the individual solutions. Those values $r_{i, j}$ form the entries of the vector $R$. Their number directly represents subcomplex $N_{1}$.

When the individual solutions are synthesised into an overall solution, more than the knowledge about the ranking is required. The compatibility of all subsolutions has to be examined as well. Five elementary ways of how to set up compatibility matrices are presented by Birkhofer (1980). The effort needed for the compatibility matrix can significantly differ depending on how the morphological box is applied. The authors performed an analysis regarding those efforts (Heller et al., 2013). Generally, it is adequate to only examine those combinations consisting of exactly two different individual solutions. With the evaluation optimised like this, the overall effort is reduced to less than half the effort for the complete evaluation. $N_{2}$ represents this compatibility assessment.

The selection of one possible solution from the set of all theoretically possible solutions costs exactly the number of evaluations expressed by the scalar values of sub-complexes $N_{1}$ and $N_{2}$. When identifying the structure of the equations for $N_{1}$ and $N_{2}$ it becomes clear, that bigger problems feature unmanageable effort. While $N_{1}$ is rising linearly with each additional sub-solution added to the morphological box, $N_{2}$ behaves factorially. That behaviour is referred to as combinatorial explosion.

\section{Mathematical Approaches}

When morphological boxes are used for generating product concepts it becomes apparent that comprehensive research will be nearly impossible with growing size of the problem. However, mathematical approaches exist to ease their application.

Exemplarily, the efforts for the box from Figure 2 are investigated. It is formed by three sub-functions (the rows) and five to three entries in the rows. It already delivers 60 different combinations. Sub-complex $N_{1}$ is 12 , resembling the number of entries in the box. Therefore, a complete assessment needs to investigate 12 elements regarding their suitability to solve the problem. The compatibility evaluation of 47 two-element chains is required (sub-complex $N_{2}$ ). It becomes clear, that with increasing sizes of the morphological box the required evaluation effort will be unmanageably high.

As a consequence, Birkhofer proposes optimisations to efficiently apply the morphological box, which were adopted by Roth later (Birkhofer, 1980), (Roth, 2000). He investigates the best size and shape of morphological boxes optimised towards minimal evaluation effort. To achieve this, he assumes given numbers of overall solutions, leading to constant numbers of elements in the morphological box. This number is called complex $N_{x}$. Leaving $N_{x}$ persistent during optimisations the ideal quantity of rows and columns can be estimated. When the 
morphological box is entirely occupied, the ideal number of rows $x$ for a minimised $N_{1}$ results in $x_{o p t, N 1}=\ln \left(N_{x}\right)$. Likewise, the ideal number of columns $k$ can be estimated to $k_{o p t, N 1}=e$ (Birkhofer, 1980). Birkhofer presents additional equations for optimised size and shape of morphological boxes for minimised effort concerning the assessment of compatibility (minimised $N_{2}$ ).

Birkhofer's investigations show that the optimal number of individual solutions is three (precisely: Euler's number $e$ ). This conclusion is valid under the premise of a constant number of overall solutions. However, when the quantity of overall solutions has to remain constant and the number of individual solutions is optimised, the quantity of sub-functions has to change. It is questionable whether this is rational or possible for actual product development although the effort would be minimal in that case. Summarising, it is arguable, if the morphological analysis intended by Zwicky can be achieved with the discussed optimisation when only three sub-solutions for each sub-function are investigated.

\section{Hierarchical Approaches}

The previous section demonstrates that the effort for evaluations within the morphological box can be estimated but not reduced. The only parameter of significant influence on the effort is the size of the morphological box. One possible answer to this challenge could be decomposing the solution field hierarchically, as shown by Weber (Weber and Condoor, 1998). Levin presents a fitting approach as Hierarchical Multicriteria Morphological Analysis (HMMA) (Levin, 1996). It can be regarded a multi-criteria optimisation. Both rankings vector $R$ and compatibility matrix are required as well. In addition, he introduces an excellence criterion for the overall solutions as vector $N$ with $N(S)=(w(S)$; $n(S))$. It reflects the two sub-complexes $N_{1}$ and $N_{2}$ (Levin, 2012). $w(S)$ is a measure for the compatibility of sub-solutions. $n(S)$ addresses the ranking of the same sub-solutions. The excellence vector $N(S)$ can be estimated for all overall solutions in $N_{x}$ enabling the identification of the most promising one. In addition, Levin suggests decomposing the problem into several sub-problems each with separate morphological analyses in order to decrease the amount of necessary evaluations. An additional analysis is needed to synthesise the results of the subproblems into the overall solution (Levin, 1996).

The HMMA approach of Levin is easy to apply and incorporates a mathematical model. Furthermore, it qualifies to handle combinatorial explosion appropriately. Nevertheless, as with conventional approaches or Birkhofer's optimisations, evaluating both ranking vector and compatibility matrix is still required for successful application.

\section{Economical Approaches}

Levin further suggests investigating the morphological field towards paretoefficiency in order to find those overall solutions that are contained in the paretooptimum set (Levin, 2012). Pareto-optimum is referred to as a state in which it is impossible to improve one aspect of an element in the set without deteriorating 
another (Wiese, 2005). Applied to the morphological box, the solution field is evaluated in order to identify those overall solutions that consist of the best individual solutions and at the same time are the most balanced ones. Only the ranking vector is required for this optimisation (Levin, 1996). Its application does not rely on the compatibility matrix in the first place. Thus, the effort for the actual evaluation is significantly reduced. However, as the compatibility is not assessed some overall solutions might not be feasible. They should be excluded from the set before conducting the evaluation a second time. The pareto-algorithm tries to find the most balanced solutions, which implies that solutions being excellent in some aspects of their technical realisation but only average or below in others might not be part of the pareto-optimum set.

\section{Additional Approaches}

In addition to hierarchical and economical approaches, Levin presents several other methods. Common with all of them is that they do not optimise evaluation effort. For HMMA with uncertainty, Levin addresses the fact that evaluation itself might be problematic due to imprecise levels of concretion with the introduction of fuzzy criteria. The number of investigations for ranking and compatibility, however, remains the same. An overview of different approaches is given in (Levin, 2006). In contrast to that, Schneider presents an approach that only evaluates a small number of representative solutions. They can be interpreted as exemplary combinations of individual solutions that represent a group of related solutions (Schneider, 2001). The approach seems to be suitable in order to reduce evaluation effort. But the chance to find new and innovative product concepts in the morphological field is reduced similarly as only a small part of the complete solution field is researched. This is directly in contrast to the initial ideas of Zwicky. Concluding, the presented approaches are not suited for dissolving the dilemma of morphological analyses. A possible way is to keep the number of functions and solutions small. Although this will lead to low effort in its application, the purpose of the morphological box gets diluted.

\section{Iterative Method for Efficient Morphological Analyses (EMA)}

To overcome this unsatisfactory state during the conceptual phase of product development projects, a new method is conceptualised that aims at supporting design engineers for an efficient application of morphological analyses. The methods are implemented in a software prototype to evaluate the effectiveness. Figure 4 displays the steps of the method for conducting efficient morphological analyses (EMA) in the early phases of product development.

The method consists of six elementary steps. The first is similar to conventional approaches and consists of the elaboration of sub-functions and individual solutions. If considered together, these two aspects, can be used to build the morphological box. The second step requires setting up the ranking of all individual solutions. This step is referred to as the prioritisation. All sub-functions 
are assessed individually regarding their suitability. Therefore, no compatibility is evaluated. This step is conducted in order to determine the first sub-complex $N_{1}$.

Figure 4. Steps of the Method for Conducting Efficient Morphological Analyses

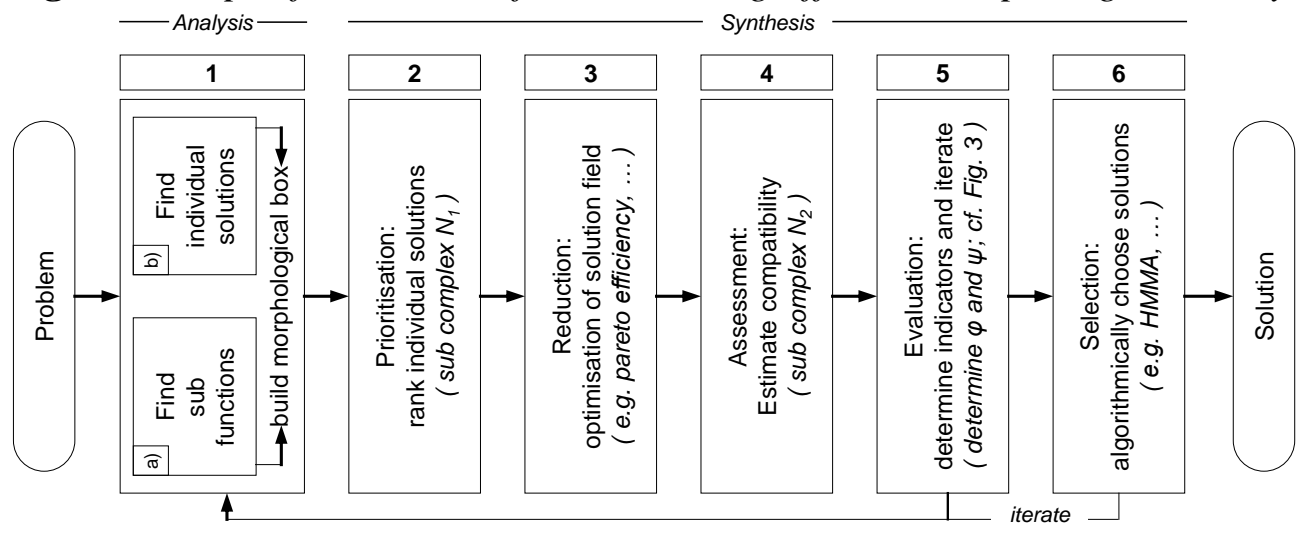

At this stage a preliminary optimisation can be executed as the third step. The algorithm used for this must only rely on the rank data, as the compatibility has not yet been assessed. Pareto-efficiency as described above can be implemented for this. Another approach is the highest rank algorithm that drops those solutions that feature a rank value below a certain threshold. The result is a set of all possible solutions that are conforming to the optimisation scheme. Admittedly, these solutions are not assessed regarding their technical compatibility. Therefore, the fourth step is introduced to overcome this limitation. The compatibility evaluation, which is now significantly reduced in its complexity due to the reduced solution field, has to be conducted regarding contradictions and benefits of combinations of two-element chains, resulting in the second sub-complex $\mathrm{N}_{2}$. As a consequence of this iterative approach, the evaluation of $N_{2}$ might result in no compatible solution. In this case, the reduction step (2) can be conducted a second time after the removal of the incompatible individual solutions. The fifth step is elementary in order to successfully conduct morphological analyses with low effort (EMA). It is installed to make sure that a certain broadness of the solution field is secured. This is achieved by determining quality indicators. The sixth step serves as the selection phase, where one or more overall solutions are picked from the result set for further realisation. Suitable algorithms for selection taking into account the two sub-complexes can be a conventional benefit analysis or more elaborated approaches like HMMA. When no appropriate solutions are retrieved, repeated cycles of the method with changed constraints are required.

\section{Establishing Quality Indicators}

This iterative approach enables the reduction of the evaluation effort significantly. Nevertheless, one fundamental problem of the efficient application of morphological analyses still remains. The risk of not investigating the total solution space because of too small morphological boxes or too similar solutions renders their application useless. To surpass this problem, the fifth step has been 
conceptualised and integrated into the method. It is installed to evaluate the solution field regarding the two main optimisation directions of the morphological approach: possible innovation as well as technical feasibility. For both directions indicator values are introduced and referred to as $\varphi$ and $\psi$. The operator estimates the indicators on a scale from 1 to 5 . Hence, they are only able to represent a subjective sight. However, they directly help to characterise the morphological field regarding both the extensiveness covered and the immediate technical feasibility. In order to obtain a high-quality solution field that contains very innovative as well as very concrete solutions, the two indicators have to hold their maximum values. Like introduced above, analyses where both indicators hold the highest value possible will lead to a required evaluation effort that is not manageable in economically reasonable applications. As Levin demonstrated, several small morphological analyses that are executed sequentially are superior to this regarding the effort. However, to minimise the risk of both omitting large parts of the solution field and only researching already known solutions, the overall quality indicator $Q$ is introduced. It is constituted by extracting the root of the product of the two quality indicators for innovation and feasibility. $\varphi_{i}$ is used to measure the potential innovation within the $i$-th iteration of the morphological analysis as estimated by the operator. Likewise, $\psi_{i}$ is used to measure the technical feasibility. Then, $Q$ is calculated after every morphological analysis before being carried over to the next iteration. At that stage, the indicator values $\varphi_{i+1}$ and $\psi_{i+1}$ are estimated again. The maximum of the $i$-th and $i+1$-th iteration is taken into account for the estimation of the quality value of that iteration $Q_{i+1}$. In the course of the separate morphological analyses, the $Q$ value has to be maximised in order to elaborate a high quality overall solution. This method directly supports engineering design methodology by exposing a single key figure that serves as a quality indicator for the morphological approach.

\section{Evaluation with Software Demonstrator}

To evaluate the effectiveness of the new method, a prototypical implementation has been conducted. Figure 5 displays the modularised structure of the prototype.

Separate applications have been conceptualised to e.g. create function structures in order to obtain the sub-function in a convenient way. Another application helps to set up the solution field using the morphological box. In addition, separate modules for the analysis incorporating the methods presented above have been implemented. One module assists the user to make his decisions in order to reduce the combinatorial variety while still being able to properly cover the solution field.

All modules are realised as standalone application realised with the Delphi software development environment in Object Pascal programming language. All data exchange within the different modules is based on human readable industry standard xml files. In addition to that, the demonstrator allows for saving all application internal object data types representing the functions, solutions, rank 
vectors and compatibility matrices as JSON object. A custom interface has been designed to implement this interface.

Figure 5. Modularised Structure of the Software Demonstrator

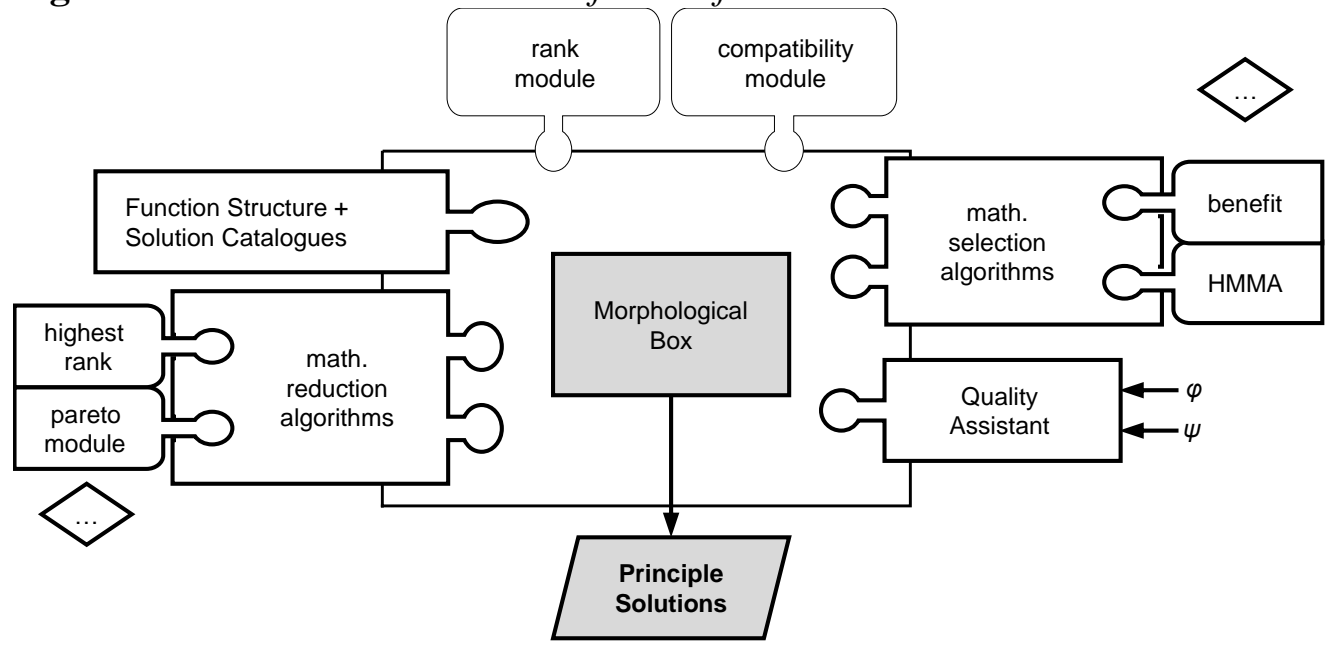

The optimisation algorithms are similarly implemented as separate units. This modularised conceptualisation enables quick adoption of new algorithms without having to revise the complete software.

The demonstrator covers the iterative method as presented in the previous section. In addition to being able to set up the solution field (the morphological field) by offering separate modules for sub-functions and corresponding solutions, the software supports the user to generate the morphological box using the matrix visualisation. Moreover, algorithms to determine the sub-complexes $N_{1}, N_{2}$ and the number of overall solutions $N_{\mathrm{x}}$ are available and displayed to the user in an information window throughout the process in order to get an impression of the implications related to the amount of generated solutions and required assessments.

Figure 6. Screenshots of the Implemented Software Prototype
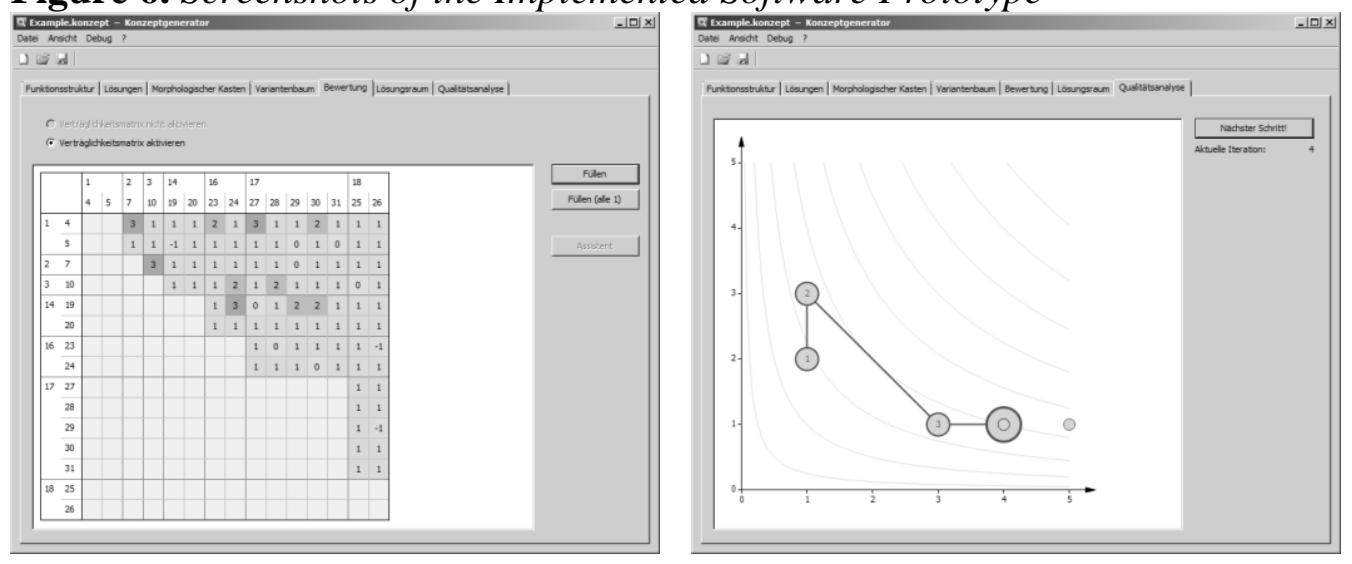
The current implementation can also handle morphological boxes that are not fully occupied and thus have different numbers of elements in each row. Besides that, algorithms to evaluate pareto-efficiency and to determine Levin's excellence vectors $N(S)$ are implemented. The Quality Assistant module has been conceptualised and integrated to support the user with key indicators regarding the extensiveness of the solution field coverage. This is achieved by implementing the two indicators $\varphi$ and $\psi$. Figure 6 on the left exemplarily shows the module for entering values for the comparisons in the compatibility matrix and on the right the user interface for the chart generated from the quality indicators for the different iterations.

\section{Exemplary Use Case}

For further evaluation, the concept generation phase for next generation multi-technology machine tools (MTPs) has been addressed with the iterative method for efficient morphological analyses (EMA). This method has been evaluated regarding its effectiveness during early phases of development processes.

One example may demonstrate the effectiveness: the generalised morphological box from Figure 2 has been filled with actual solutions for three exemplary sub-problems: plunger geometry, force creation method and construction layout. The morphological box is given in Figure 7 . Three to five individual solutions have been researched for each function. The systematic combination of those twelve elements $\left(N_{1}\right)$ results in as many as 60 different overall solutions $\left(N_{\mathrm{x}}\right)$. As detailed above, the compatibility evaluation effort can be determined to 47 investigations of combinations of two-element chains $\left(N_{2}\right)$. The overall effort thus can be estimated as 59 separate investigations of the individual solutions or combinations.

Figure 7. Morphological Box Investigated in Exemplary Use Case

\begin{tabular}{|c|c|c|c|c|c|}
\hline & 1 & 2 & 3 & 4 & 5 \\
\hline $\begin{array}{c}\text { Sub-Problem 1: } \\
\text { plunger geometry }\end{array}$ & Round & $\begin{array}{c}\text { Prismatic / } \\
\text { Flat }\end{array}$ & Prism (O) & Prism (X) & $\begin{array}{c}\text { Dove } \\
\text { Tail }\end{array}$ \\
\hline $\begin{array}{c}\text { Sub-Problem 2: } \\
\text { force creation }\end{array}$ & Spindle & Crank shaft & $\begin{array}{c}\text { Toggle } \\
\text { Lever/Joint }\end{array}$ & Hydraulics & \\
\hline $\begin{array}{c}\text { Sub-Problem 3: } \\
\text { construction } \\
\text { layout }\end{array}$ & $\begin{array}{c}\text { Single } \\
\text { Column } \\
\text { ("C" / } \\
\text { Open) }\end{array}$ & $\begin{array}{c}\text { Two } \\
\text { Column } \\
\text { ("C"C/ } \\
\text { Open) }\end{array}$ & $\begin{array}{c}\text { Double } \\
\text { Column } \\
\text { ("O"O/ } \\
\text { Closed) }\end{array}$ & & \\
\hline
\end{tabular}

In this example, the potential innovation indicator $\varphi$ for the first iteration run has been assessed with a value of 1 as the solution field has been constituted with rather conservative entries. Opposed to that, the feasibility indicator $\psi$ was evaluated with a value of 5 as all entries already have been realised in products. Detailed discussion of the solutions itself is omitted because the results in general 
heavily depend on the engineering and production capabilities of the manufacturer. Nevertheless, ranking and compatibility matrix have been filled with randomised values that reflect typical manufacturing restrictions. The solution space could successfully be limited to only six remaining overall solutions while just using the rank vector. The reduction of the solution space can be considered significant $(90 \%)$ and the effort for the actual compatibility assessment could be reduced by $12.7 \%$.

\section{Summary and Outlook}

The review of the presented approaches consistently shows that the application of morphological analyses implies an unmanageable effort if conducted in the originally intended way. Existing optimisation approaches do not change this fact. Hence, industrial application remains challenging. A new method that iteratively covers parts of the total solution field with the help of a quality measure was introduced. The underlying methods were detailed and implemented in a software prototype. The evaluation in a case study for MTP conceptualisation has been presented.

The topic addressed here is subject to current research at RWTH Aachen University. Evaluating the effectiveness of the method in more industrial projects and educational applications will be the next step. In addition, incorporating design and manufacturing context information for every entry of the morphological box will be investigated in order to automatically determine the indicator values for the quality assessment measure.

\section{Acknowledgements}

The authors would like to thank the German Research Foundation DFG for the support of the depicted research within the Cluster of Excellence "Integrative Production Technology for High-Wage Countries".

\section{References}

Bernhardt, R. (1981). Systematisierung des Konstruktionsprozesses [Systematization of the design process]. Düsseldorf: VDI-Verlag.

Birkhofer, H. (1980). Analyse und Synthese der Funktionen technischer Produkte [Analysis and synthesis of the functions of technical products]. Thesis. FortschrittsBerichte der VDI-Zeitschriften: Vol. 70. Düsseldorf: VDI-Verlag.

Franke, H. J (1999). Ungelöste Probleme der Konstruktionsmethodik [Unsolved problems in design methodology]. In: Franke, H. J. (Ed.), Konstruktionsmethodik - quo vadis. Symposium anläßlich des 80. Geburtstags von Professor Dr.-Ing. Karlheinz Roth. Aachen: Shaker, 13-30. 
Koller, R. (1998). Konstruktionslehre für den Maschinenbau: Grundlagen zur Neu- und Weiterentwicklung techn. Produkte [Design for engineering: foundations for new and further development techn. Products]. 4th ed. Berlin: Springer.

Koller, R., Kastrup, N. (1998). Prinziplösungen zur Konstruktion technischer Produkte [Principle solutions for the construction of technical products]. 2nd ed. Berlin: Springer.

Levin, M. S. (1996). Hierarchical Morphological Multicriteria Design of Decomposable Systems. In Concurrent Engineering, 4(2), 111-117.

Levin, M. S. (2006). Composite Systems Decisions. London: Springer.

Levin, M. S. (2012). Morphological methods for design of modular systems (a survey). Computing Research Respository, abs/1201.1712.

Pahl, G., Beitz, W., Feldhusen, J., Grote, K.-H. (2007). Engineering Design: A Systematic Approach. London: Springer.

Pine, B. J. (1993). Mass customization: The new frontier in business competition. Boston: Harvard Business.

Roth, K. (2000). Konstruieren mit Konstruktionskatalogen [Designing with design catalogs]. 3rd ed. Vol. 1. Berlin: Springer.

Schneider, M. (2001). Methodeneinsatz in der Produktentwicklungs-Praxis: Empirische Analyse, Modellierung, Optimierung und Erprobung [Methods used in the product development practice: Empirical analysis, modeling, optimization and testing]. Düsseldorf: VDI-Verlag.

Tomiyama, T., Gu, P., Jin, Y., Lutters, D., Kind, C., Kimura, F. (2009). Design methodologies: Industrial and educational applications". CIRP - Manufacturing Technology. 58(2), 543-565.

VDI Guideline 2221 (1993). Methodik zum Entwickeln und Konstruieren technischer Systeme und Produkte [Approach to the development and design of technical systems and products]. Berlin: Beuth.

VDI Guideline 2727 (2010). Konstruktionskataloge: Lösung von Bewegungsaufgaben mit Getrieben [Catalogues for machine design: solution of motion tasks with gears]. Berlin: Beuth.

Weber, R. G., \& Condoor, S. S. (1998). Conceptual design using a synergistically compatible morphological matrix. In: Frontiers in Education Conference, 1998. FIE '98. 28th Annual, (1) 171-176.

Wheelwright, S. C., Clark, K. B. (1992). Revolutionizing product development: Quantum leaps in speed, efficiency, and quality. New York: Free Press.

Wiese, H. (2005). Mikroökonomik: Eine Einführung in 379 Aufgaben [Microeconomics: An introduction to 379 tasks]. 4th ed. Berlin: Springer.

Zwicky, F. (1947). Morphology and Nomenclature of Jet Engines. Aeronautical Eng. Review. 6(6), 49-50.

Zwicky, F. (1949). Morphologische Forschung [Morphological research]. Pasadena. 